\title{
A Randomised Prospective Study Analysing the Effects of Lowering Serum Uric Acid in Chronic Kidney Disease (CKD) Progression and Cardiovascular Outcomes
}

\author{
Gupta A, Choudhary SS, Pursnani L*, Mahapatra H and Verma H \\ Department of Nephrology, Dr. Ram Manohar Lohia Hospital \& Post 1Graduate \\ Institute of Medical Education and Research, India
}

*Corresponding author: Lalit Pursnani, Department of Nephrology, Dr. Ram Manohar

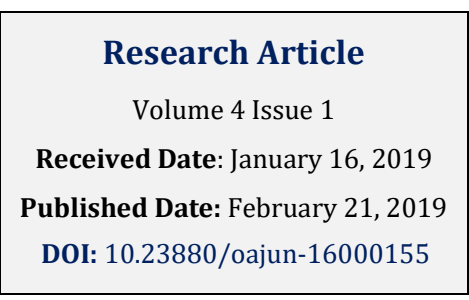

Lohia Hospital \& Post Graduate Institute of Medical Education and Research, New Delhi, India, Tel: 9958268930; Email: drlalitkpursnani@gmail.com

\section{Abstract}

Uric acid is an independent risk factor for the development and progression of kidney disease. In last two decades number of observational studies has examined the potential link between management of hyperuricemia with CKD progression, having conflicted results. Hence, this prospective, randomised controlled study was conducted among stage 3-5 CKD patients in patients to evaluate the effect of lowering uric acid levels in reduction of hypertension, and kidney disease progression and cardiovascular risk. Patients were randomized into two groups, one group (n=100) receiving allopurinol with a dose of 100-300 mg/day plus standard treatment and other group (n=50) receiving only standard treatment. Patients were followed at 3, 6 and 12 months of treatment. After 12 months of allopurinol treatment, serum uric acid levels were significantly $(\mathrm{p}<0.0001)$ lowered $(8.095 \pm 0.877 \mathrm{mg} / \mathrm{dL}$ to $6.977 \pm 0.569 \mathrm{mg} / \mathrm{dL}) \mathrm{in} \mathrm{patients}$ receiving allopurinol, whereas, the uric acid levels were significantly ( $\mathrm{p}<0.0001)$ increased in control group $(7.910 \pm$ $0.701 \mathrm{mg} / \mathrm{dL}$ to $8.196 \pm .741 \mathrm{mg} / \mathrm{dL}$ ). A decrease in estimated glomerular filtration rate (eGFR) was observed at the end of 12 months in both the groups, but the decrease in eGFR was significantly more in the control group as compared to the allopurinol group $(\mathrm{P}<0.0001)$. There was no significant effect of allopurinol on cardiovascular outcomes. This study evaluated that lowering of serum uric acid was effective in slowing the renal disease progression and cardiovascular risk in patients with CKD as compared to the controls. However, no significant beneficial effect of allopurinol treatment was observed on hypertension and cardiovascular outcomes in these patients.

Keywords: Chronic kidney disease; Uric acid; Hypertension; Cardiovascular risk; Allopurinol treatment

Abbreviations: CKD: Chronic Kidney Disease, UA: Uric Acid, eGFR: Estimated Glucose Filtration Rate, SBP:
Systolic Blood Pressure, DBP: Diastolic Blood Pressure, NYHA: New York Heart Association, CHF: Congestive 
Heart Failure, BP: Blood Pressure, ARIC: Atherosclerosis Risk in Communities: MDRD: Modification of the Diet in Renal Disease.

\section{Introduction}

Uric acid (UA) is the end product of purine metabolism in humans. Hyperuricemia is common in kidney disease due to decreased UA clearance [1]. The mechanism of kidney damage from UA probably includes induction of afferent arteriopathy, inflammation, and activation of the renin-angiotensin system [2]. The mechanism leading from elevation of UA level to chronic kidney disease (CKD) may be direct or may be mediated through other risk factors. Hyperuricemia is proved to be one of the causes of hypertension and insulin resistance [3], and these risk factors may lead to kidney disease. Previously, it was a hallmark for kidney damage and also was associated with kidney disease development and progression [4]. However, recent studies have showed that serum UA levels may have role in hypertension, renal, cardiovascular diseases [5].

There are currently various epidemiological studies that point to the role of UA as an independent risk factor for the development and progression of kidney disease, but without definitive conclusions. In experimental models, allopurinol prevented vascular complications through its antioxidant effects by blocking the generation of free radicals. In an experimental model, hyperuricemic rats with elevated blood pressure were treated with either xanthine oxidase inhibitor (allopurinol) or uricosuric agent (benziodarone) which showed reduction in the levels of UA. The serum UA levels were directly associated with the blood pressure [6]. Recently, one study has also reported allopurinol can reduce left ventricular hypertrophy and improve endothelial function in CKD patients [7]. Similarly, another prospective, randomised study consisting of 113 patients with estimated glucose filtration rate (eGFR) $<60 \mathrm{~mL} / \mathrm{min}$ and treated with allopurinol in one group and standard therapy with other. After 24 months of treatment, the allopurinol slows the progression of renal disease and reduces cardiovascular and hospitalization risk in these patients [8].

However, in India, there few studies evaluating the effect of UA lowering therapy in CKD progression. Recently, a study has been published by Nizams Institute of Medical Sciences, Hyderabad; Andhra Pradesh, India in which they evaluated the role of allopurinol for prevention of progression of kidney disease with hyperuricemia [9]. This was a retrospective cohort study in chronic kidney disease patients with hyperuricemia from 1998 to 2008. An inverse correlation was noted between serum UA levels and the eGFR at 6 months, 1 year, and 2 years of treatment. Also, allopurinol treatment reduces UA levels in blood and progression of renal disease in CKD patients with hyperuricemia. It was a retrospective observational study which did not evaluate the cardiovascular outcomes o flowering UA in these patients. There sults might be limited by the concomitant use of statins, antiplatelet and renin angiotensin aldosterone system blocker drugs. To the best of our knowledge, in India, this is the first randomised, prospective study evaluating the effect of lowering UA levels on cardiovascular outcomes in chronic kidney disease patients.

As a growing body of evidence links high UA levels with the development of hypertension, CKD, ESRD, cardiovascular events, and death, we undertook this randomised controlled study with aim to analyse the effect of lowering UA in patients with CKD (stage 3-5) in slowing of renal disease progression and reduction of hypertension and cardiovascular outcome.

\section{Materials and Methods}

\section{Patient's Population}

This was a prospective randomised study conducted at tertiary teaching care hospital during the period from January 2013 to April 2014. Patients with presence of renal disease, defined as having an estimated GFR (eGFR) lower than $60 \mathrm{ml} / \mathrm{min}$, stable clinical condition in terms of no hospitalizations or cardiovascular events within the 3 months before screening; and serum UA $>5.6 \mathrm{mg} / \mathrm{dl}$ in females and $>7 \mathrm{mg} / \mathrm{dl}$ in males were included in the study.

The exclusion criteria included the patients who did not provide consent, age $<18$ years, those who had current treatment or previous hypersensitivity/intolerance to allopurinol, 2 or more episodes of gout in past 6 months, active infections or serum UA level $>13 \mathrm{mg} / \mathrm{dl}$ in men and $>10 \mathrm{mg} / \mathrm{dl}$ in women, history of UA calculi. Patients with pregnancy or breast feeding, end stage renal disease or on concurrent treatment with immunosuppressive drugs were also excluded from study. The study protocol was approved by Institutional Ethics Committee. Informed consent form was obtained from participants before enrolling into the study. 


\section{Randomization and Intervention}

Patients were randomly assigned into two groups. One group received allopurinol 100-300 mg/day (adjusted according to eGFR) in addition to standard management of CKD, while the other group continued with the standard management. Randomization was conducted by simple randomization method and allocation concealment was implemented by envelope technique.

\section{Outcome Analysis and Follow-up}

Initial assessment of cardiovascular risk factors in patients was done at beginning of the study. Hypertension was defined as systolic blood pressure (SBP) $>140 \mathrm{~mm}$ $\mathrm{Hg}$ or diastolic BP (DBP) $>90 \mathrm{~mm} \mathrm{Hg}$ on repeated measurements or the use of antihypertensive drugs. The arterial blood pressure was measured three times after a 15-min resting period and mean values were calculated for SBP and DBP for all participants. eGFR was estimated by Modification of the Diet in Renal Disease (MDRD)-4 equation and was carefully recorded during follow-up assessment.

Cardiovascular event was considered if the patient had a myocardial infarction, coronary revascularization, or angina pectoris. All symptomatic patients with New York Heart Association (NYHA) class II to IV dyspnoea were subjected to chest x-ray examination and echocardiogram to assess left ventricular function. Congestive heart failure (CHF) was defined as ejection fraction <45\% [10]. Occurrence of any congestive heart failure, cerebrovascular accidents or peripheral vascular disease during the period of follow-up were also taken into account and included in cardiovascular events.

Clinical, biochemical, and inflammatory parameters were measured at baseline, 3,6 , and 12 months of treatment. During each follow-up session, SBP and DBP were recorded, and daily urinary protein excretion, haemoglobin level, white blood cell count, platelet count, serum $\mathrm{Cr}$ level, alanine aminotransferase level, fasting total cholesterol level, low-density lipoprotein cholesterol level, high-density lipoprotein cholesterol level, triglyceride level, serum UA level, and high sensitivity Creactive protein level were checked.

\section{Statistical Analysis}

Data was analysed by SPSS Statistics 19 of windows 7 . Standard statistical tools were used to analyse the data. The data was double entered and checked for any discrepancies. Values were expressed as mean \pm SD, mean \pm SEM, or median to study samples in two categories separately. Students T-test or Chi-square test was used to study the correlation of treatment with allopurinol on the progression of CKD. Analysis of variance test was used when parameters of more than two groups were compared. Pearson's correlation coefficient was calculated to analyse correlation between two parameters. Cox proportional hazard models were used to evaluate the risk of cardiovascular events, adjusted for several groups of covariates. The $P$ value less than 0.05 was considered to be statistically significant.

\section{Results}

A total of 150 patients fulfilling the inclusion criteria were enrolled in the study. One hundred patients [59 males (59\%), 41females (41\%)] were randomised to the allopurinol group and 50 patients [27 males (54\%), 23 females (46\%)] to the control group (Table 1\&2).

In the allopurinol group the primary kidney diseases were diabetic nephropathy (30\%), chronic interstitial nephritis (23\%), chronic glomerulonephritis (16\%), hypertensive nephropathy $(15 \%)$, obstructive uropathy (1\%), autosomal polycystic kidney disease $(6 \%)$ and others $(9 \%)$. In the control group the primary kidney diseases were diabetic nephropathy $(30 \%)$, chronic interstitial nephritis (26\%), chronic glomerulonephritis (18\%), hypertensive nephropathy (12\%), obstructive uropathy (2\%), autosomal polycystic kidney disease (4\%) and others $(8 \%)$.

\begin{tabular}{|c|c|c|c|}
\hline Parameters & Allopurinol Group (n=100) & Control Group (n=50) & P value \\
\hline Age (years) & $42.67(13.902)$ & $43.84(12.831)$ & 0.619 \\
\hline Sex, Male: Female & 1.439 & 1.173 & 0.341 \\
\hline Hemoglobin (g/dL) & $11.42(0.89)$ & $11.48(0.49)$ & 0.664 \\
\hline Serum creatinine (mg/dl) & $2.28(0.83)$ & $2.15(0.70)$ & 0.339 \\
\hline eGFR (ml/min per 1.73 m2) & $34.47(12.50)$ & $35.10(12.12)$ & 0.768 \\
\hline Serum uric acid (mg/dL) & $8.09(0.87)$ & $7.91(0.70)$ & 0.195 \\
\hline Serum hs-CRP (mg/L) & $6.89(1.72)$ & $6.63(1.05)$ & 0.335 \\
\hline ESR (mm/hr) & $24.46(7.65)$ & $22.16(5.37)$ & 0.059 \\
\hline
\end{tabular}




\section{Open Access Journal of Urology \& Nephrology}

\begin{tabular}{|c|c|c|c|}
\hline Serum Albumin (g/dL) & $3.50(0.21)$ & $3.51(0.18)$ & 0.847 \\
\hline Albuminuria (mg/dL) & $379.7(32.5)^{*}$ & $392.62(55)^{*}$ & 0.917 \\
\hline Total cholesterol (mg/dL) & $161.24(15.54)$ & $159.64(13.57)$ & 0.537 \\
\hline Systolic BP (mmHg) & $140.56(15.51)$ & $139.88(16.56)$ & 0.805 \\
\hline Diastolic BP (mmHg) & $85.10(6.67)$ & $84.08(7.417)$ & 0.397 \\
\hline
\end{tabular}

Data presented as a mean \pm SD or median*. BP, blood pressure; eGFR, estimated glomerular filtrate rate; ESR, erythrocyte sedimentation rate; hs CRP, high sensitivity C-reactive protein.

Table 1: Baseline demographic and biochemical profile of patients in allopurinol group and control group

\begin{tabular}{|c|c|c|c|}
\hline Characteristic \% (n) & Allopurinol Group (n=100), \% (n) & Control Group (n=50), \% (n) & P value \\
\hline Diabetes Nephropathy & $30(30)$ & $30(15)$ & $\mathrm{ns}$ \\
\hline Chronic interstitial disease & $23(23)$ & $26(13)$ & $\mathrm{ns}$ \\
\hline hronic glomerulonephriti & $16(16)$ & $18(9)$ & $\mathrm{ns}$ \\
\hline Hypertensive nephropathy & $15(15)$ & $12(6)$ & $\mathrm{ns}$ \\
\hline Obstructive Uropathy & $1(1)$ & $4(2)$ & $\mathrm{ns}$ \\
\hline $\begin{array}{c}\text { Autosomal Polycystic } \\
\text { Kidney Disease }\end{array}$ & $6(6)$ & $8(4)$ & $\mathrm{ns}$ \\
\hline Other etiologies & $9(9)$ & $62(31)$ & $\mathrm{ns}$ \\
\hline Calcium channel blockers & $62(62)$ & $24(12)$ & $\mathrm{ns}$ \\
\hline Beta Blockers & $20(20)$ & $38(19)$ & $\mathrm{ns}$ \\
\hline RAAS blockers & $41(41)$ & $16(8)$ & $\mathrm{ns}$ \\
\hline Anti-platelets & $20(20)$ & $22(11)$ & $\mathrm{ns}$ \\
\hline Statins & $25(25)$ & $48(24)$ & $\mathrm{ns}$ \\
\hline Diuretics & $52(52)$ & $34(17)$ & $\mathrm{ns}$ \\
\hline $\begin{array}{c}\text { 2 antihypertensives } \\
\text { 3 or more }\end{array}$ & $33(33)$ & $10(5)$ & $\mathrm{ns}$ \\
\hline No. of Hypertensive & $12(12)$ & $72(36)$ & \\
\hline
\end{tabular}

Table 2: Baseline characteristics of patients in allopurinol group and control group.

\section{Blood Pressure Control}

In the allopurinol group, 75 patients $(75 \%)$ had hypertension and 36 patients $(72 \%)$ were hypertensive in the control group. Mean systolic blood pressure (BP) was $140.56 \pm 15.51 \mathrm{mmHg}$ and mean diastolic BP was $85.10 \pm$ $6.67 \mathrm{~mm} \mathrm{Hg}$ in the allopurinol group. Likewise, in the control group, mean systolic blood pressure (BP) was $139.88 \pm 16.56 \mathrm{~mm} \mathrm{Hg}$ and mean diastolic $\mathrm{BP}$ was $84.08 \pm$ $7.417 \mathrm{~mm} \mathrm{Hg}$. All hypertensive patients were put on appropriate anti-hypertensive therapy. In the treatment group, 33\% patients were on 2 anti-hypertensives and 12\% patients were on 3 or more anti-hypertensives. Similarly in the control group $34 \%$ patients were on 2 antihypertensive and $10 \%$ patients were on 3 or more antihypertensives. Mean baseline BP was comparable between the two groups and there was no significant difference in systolic or diastolic BP between the two groups at 3, 6 and 12 months of follow-up. Though, the proportion of patients on 3 or more antihypertensives was more in control group at the end of the study $(13 \%$ in control group vs. $11 \%$ in allopurinol group), this difference didn't reach the statistical significance.

\section{Biochemical Parameters Analysis within the Groups}

After 12 months of allopurinol treatment, serum UA levels were significantly decreased in subjects treated with allopurinol, from $8.095 \pm 0.877 \mathrm{mg} / \mathrm{dl}$ to 6.977 $\pm .569 \mathrm{mg} / \mathrm{dl} \quad(\mathrm{P}<0.0001)$, whereas serum UA levels increased in subjects with the control group over a period of 12 months from $7.910 \pm 0.701 \mathrm{mg} / \mathrm{dl}$ to $8.196 \pm 0.741$ $\mathrm{mg} / \mathrm{dl}(\mathrm{P}<0.0001)$.

\section{Comparison between the Groups}

There was a decrease of $1.11 \pm 0.70 \mathrm{mg} / \mathrm{dl}$ in mean UA level in the allopurinol group, whereas there was an increase of $0.286 \pm 0.29 \mathrm{mg} / \mathrm{dl}$ in the control group at the end of the 12 months. There was a significant difference between changes in UA levels from baseline when two 
groups were compared ( $\mathrm{P}<0.0001$ at 3,6 and 12 months). Mean value of serum creatinine increased from $2.28 \pm$ $0.83 \mathrm{mg} / \mathrm{dl}$ to $2.38 \pm 0.90 \mathrm{mg} / \mathrm{dl}$ at 12 months in allopurinol group and from $2.15 \pm 0.70 \mathrm{mg} / \mathrm{dl}$ to $2.34 \pm$ $0.76 \mathrm{mg} / \mathrm{dl}$ in the control group $(\mathrm{P}<0.0001$ for change of creatinine at 12 months between the groups). There was no significant difference in mean values of hemoglobin, total cholesterol, LDL cholesterol and serum albumin in either group throughout the study.

\section{Progression of Renal Disease Analysis within the Groups}

There was a fall in eGFR (MDRD-4) observed in both the groups by the end of the study. In the allopurinol group eGFR decreased from $34.47 \pm 12.50 \mathrm{ml} / \mathrm{min}$. per $1.73 \mathrm{~m} 2$ at baseline to $32.90 \pm 12.51 \mathrm{ml} / \mathrm{min}$. per 1.73 m2at 12 months $(\mathrm{P}<0.0001$ in comparison to baseline values), whereas in the control group, it decreased from $35.10 \pm 12.12 \mathrm{ml} / \mathrm{min}$. per $1.73 \mathrm{~m} 2$ at baseline to $31.55 \pm$ $10.86 \mathrm{ml} / \mathrm{min}$. per $1.73 \mathrm{~m} 2$ at 12 months $(\mathrm{P}<0.0001$ in comparison to baseline values). In the allopurinol group, the change in eGFR from baseline was insignificant at 3 months but it came out to be significant at the end of the study.

\section{Comparison between the Groups}

The change in mean eGFR during follow-up at 3, 6 and 12 months as compared to the baseline and a comparison of this change between the 2 groups was analysed. In the control group, eGFR decreased $3.55 \pm 2.50 \mathrm{ml} / \mathrm{min}$ per $1.73 \mathrm{~m} 2$, and in allopurinol group, eGFR decreased $1.57 \pm$ 2.85 per $1.73 \mathrm{~m} 2$ after 12 months $(\mathrm{P}<0.0001$ between the groups).

\section{Correlation between Change in eGFR (CKD Progression) and UA}

A decrease in eGFR was observed at the end of 12 months in both the groups, but the decrease in eGFR was significantly more in the control group as compared to the allopurinol group $(\mathrm{P}<0.0001)$ as shown in figure 1.

1. Another important observation was that serum UA decreased significantly in allopurinol group, whereas it increased in the control group $(\mathrm{P}<0.0001)$.

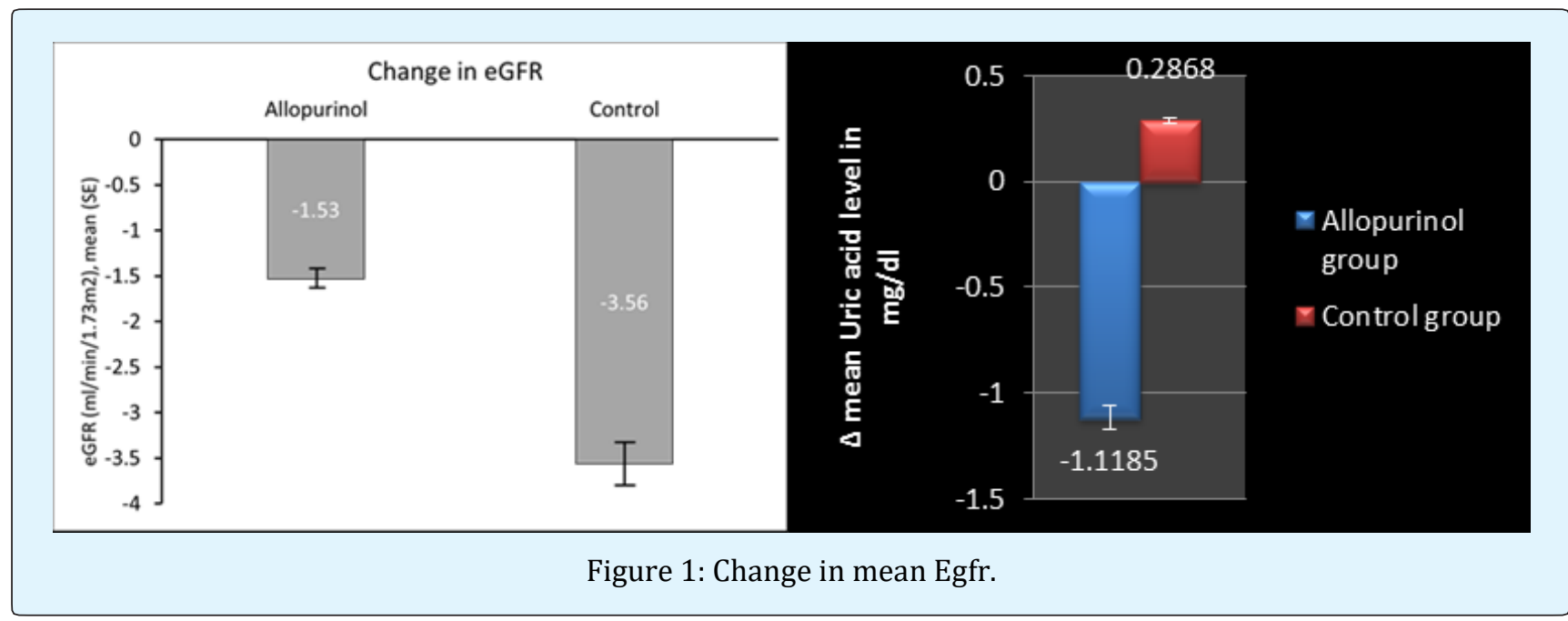

The correlation between change in eGFR and change in serum UA was evaluated in patients with control group. An inverse correlation was observed between changes in eGFR and changes in serum UA in patients who didn't receive allopurinol intervention. This was consistent at 3 , 6 and 12 months of follow-up.

\section{Cardiovascular Events and Deaths}

In the allopurinol group $18(18 \%)$ patients had cardiovascular co-morbidity. Eight patients had ischemic coronary events, four patients had congestive heart failure and six patients had cerebro-vascular accidents. In the control arm four patients had cardiovascular events, ischemic heart disease $(\mathrm{n}=3)$ and cerebro-vascular accidents $(n=1)$. No deaths were recorded in either group during the follow-up period. Two patients required dialysis at the end of the study, one from the allopurinol group and other from the control group. After 12 months of follow-up period, 19 patients suffered from cardiovascular event including 11 from allopurinol group and 8 from control group. Among these 19 patients, 15 were diabetic and 12 were hypertensive. The 
cardiovascular events were ischemic coronary events $(n=6)$, congestive heart failures $(n=10)$, cerebrovascular accidents $(n=3)$. All received appropriate therapy based on good clinical practices.
Kaplan-Meier survival showed that patients in the allopurinol group had lower cardiovascular risk than patients in the control group but this difference didn't reach the statistical significance (Figure 2).

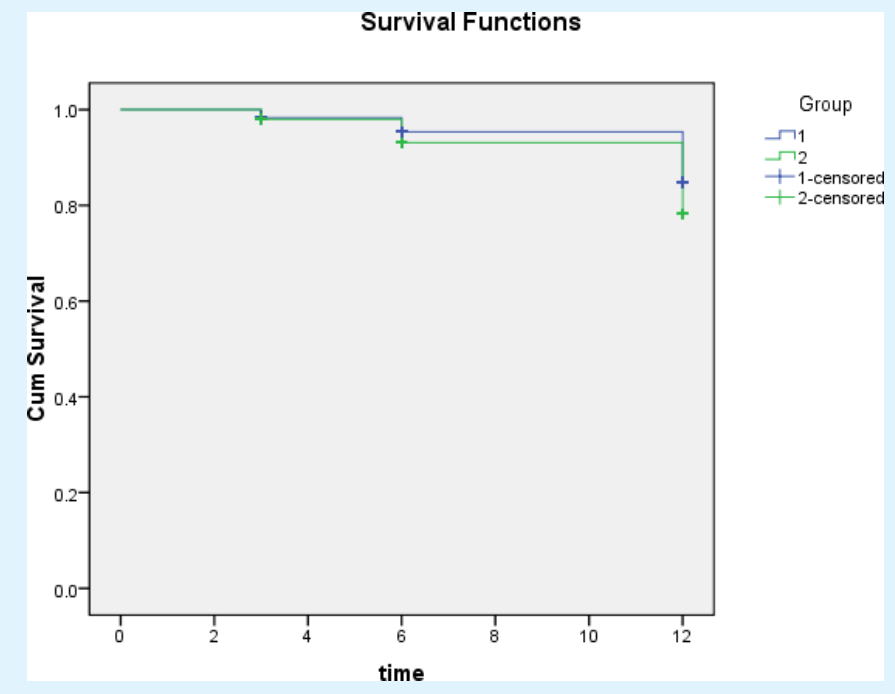

Figure 2: Effect of allopurinol treatment in cardiovascular events. (Log rank: 1.42; $\mathrm{P}=0.233$ ).

\section{Cox- Regression Analysis for Cardiovascular Events}

Regression cox analysis adjusted for age, eGFR change, $\mathrm{DM}$, previous CAD, and UA levels showed that high hs-CRP levels increased the risk of cardiovascular events while there was no significant impact of allopurinol treatment on cardiovascular events (Table 3).

\begin{tabular}{|c|c|c|c|c|c|c|c|c|}
\hline & \multicolumn{9}{|c|}{} & \multicolumn{2}{c|}{ 95.0\% CI for Exp (B) } \\
\cline { 2 - 9 } & B & SE & Wald & df & Sig. & Exp(B) & Lower & Upper \\
\hline hs-CRP & 0.533 & 0.159 & 11.287 & 1 & 0.001 & 1.703 & 1.248 & 2.324 \\
\hline Group & 0.018 & 0.382 & 0.002 & 1 & 0.962 & 1.019 & 0.482 & 2.153 \\
\hline
\end{tabular}

Table 3: Cox regression analysis showing risk of new cardiovascular events.

The hazard ratio of hs-CRP for cardiovascular events was 1.703 implicating $70 \%$ increased risk for cardiovascular events in patients with high hs-CRP levels (higher than mean for all cases i.e. $6.485 \mathrm{mg} / \mathrm{L}$ ) which was found to be significant $(\mathrm{P}=0.001)$.

\section{Discussion}

In the present study, we randomised the patients into two groups. One group of 50 patients received standard therapy for CKD, while other group of 100 patients received allopurinol 100-300 $\mathrm{mg} /$ day (dose adjusted according to eGFR) in addition to the standard therapy for CKD patients. The baseline characteristics of patients in the 2 groups were comparable and no significant difference was observed between the different baseline variables.

\section{Allopurinol Treatment and Hypertension}

Hyperuricemia has been related to high blood pressure. This is thought to be mediated through activation of the renin-angiotensin system, either directly, by decreasing neuronal nitric oxide synthase in the juxtaglomerular apparatus, or indirectly, through decreasing renal perfusion by stimulating the afferent arteriolar vascular smooth cell proliferation or through the induction of cyclo-oxygenase-2 in the macula densa 
and arterioles.2,6 To our knowledge there is only one study which demonstrated the antihypertensive effect of allopurinol in adolescent patients with initial hypertension [11]. In the present study, although we showed that BP decreased in the allopurinol group $(140.56 \pm 15.51 / 85.10 \pm 6.67 \mathrm{mmHg}$ at baseline and $134.98 \pm 9.83 / 83.04 \pm 5.37 \mathrm{mmHg}$ at the end of the study), it was not significantly different from the control group $(\mathrm{P}=0.910,0.533)$. The proportion of patients maintaining blood pressure on 3 or more antihypertensives (as compared to baseline) was higher in the control group but it was not statistically significant.

These results were similar to the study of 113 patients by Goicoechea, et al. which evaluated the effect of allopurinol on hypertension and other cardiovascular risk factors in CKD patients. There may be two main reasons for this observation. At the time of study participation most of the patients had history of long-term hypertension and all the patients had confirmed renal diseases with impaired renal function. This may have caused damage to the arteries and kidneys, and the hypertension could have been the result of multiple factors. Hence, allopurinol treatment alone couldn't affect BP control to a significant extent. Secondly, the concomitant use of antihypertensives and dietary advice to all patients in the study population intended to maintain normal blood pressure might have led to similar $\mathrm{BP}$ control in both the group.

\section{Allopurinol Treatment and Progression of CKD}

There are several observational studies demonstrating the possible correlation between serum UA levels and the progression of CKD; however, the results were conflicting. In view of these conflicting results, the most appropriate method to evaluate the involvement of UA in the pathogenesis of CKD progression is to determine whether lowering UA levels slows the rate of renal disease progression. In this direction, Siu, et al. studied the effect of the lowering of serum UA levels on progression of CKD in an open-label RCT of allopurinol therapy (100-300 $\mathrm{mg} / \mathrm{d}$ ) for 12 months in 54 patients [12]. They concluded allopurinol therapy significantly decreases serum UA levels in hyperuricemic patients with mild to moderate chronic kidney disease. Allopurinol was well tolerated and may contribute in maintaining renal function during 12 months of therapy. Similarly, Goicoechea, et al. randomized 113 patients with CKD to allopurinol $100 \mathrm{mg}$ daily $(n=57)$ or no study medication $(n=56)$. After 24 months, eGFR decreased by $3.3 \mathrm{ml} / \mathrm{min} / 1.73 \mathrm{~m}^{2}$ in the control group and increased by $1.3 \mathrm{ml} / \mathrm{min} / 1.73 \mathrm{~m}^{2}$ in the allopurinol group ( $\mathrm{P}=0.018)$.
In our study, we demonstrated that allopurinol is able to slow the progression of renal disease after a follow-up of 12 months. In the control group, eGFR decreased 3.55 $\mathrm{ml} / \mathrm{min} / 1.73 \mathrm{~m}^{2}$, and in allopurinol group, eGFR decreased $1.57 \mathrm{ml} / \mathrm{min} / 1.73 \mathrm{~m}^{2}$ after 12 months $(\mathrm{P}<0.0001$ between the groups). The baseline characteristics like age, sex ratio, primary kidney disease, degree of hypertension, serum UA levels, hs-CRP levels, albuminuria and use of antihypertensives were similar in both groups.

We observed a decrease in eGFR in both the groups, however, in Goicoechea, et al. study, eGFR increased in the allopurinol group by the end of the study. Though in this study, the decrease in eGFR was significantly less in the allopurinol group as compared to the control group. Serum UA levels decreased significantly in the allopurinol group whereas it increased in the control group. By the end of the study, a significant inverse correlation was found between serum UA levels and eGFR in the control group. Hence, the beneficial effect of allopurinol slowing down the progression of renal disease could be related to the decrease of UA levels.

\section{Allopurinol Treatment and Cardiovascular Risk}

The role of UA as an independent cardiovascular risk factor is controversial. Sub analysis of the Atherosclerosis Risk in Communities (ARIC) has demonstrated that hyperuricemia is associated with insulin resistance and mortality in the non-CKD population [13]. In another report when the use of diuretics was adjusted, no correlation was found between UA and cardiovascular disease [14]. A few small, short-term, single-Centre studies have indicated that xanthine oxidase inhibitors may exert favourable effects on cardiovascular risk factors and surrogate outcome measures. In this study, we did not find any significant beneficial effect of allopurinol treatment on cardiovascular outcomes in CKD patients. In cox regression analysis adjusted for age, gender, serum UA and GFR change; only hs-CRP had a significant effect on cardiovascular outcomes $(\mathrm{HR}=1.703, \mathrm{P}=0.001)$.

No serious adverse effects related to allopurinol were reported. Majority of the studies evaluated the effect of allopurinol on CKD progression, showing the efficient and safe use of allopurinol in patients with CKD with dose adjusted eGFR. However, serious reactions like StevensJohnson syndrome have also been reported with allopurinol. Hence, it should be used with precaution in patients with history of drug allergies. 
There were some limitations to the present study. It was not designed in a double-blinded fashion; the patients were followed for a relatively shorter period of time and the potential role of dietary factors in the results was not evaluated.

\section{Conclusion}

In conclusion lowering of serum UA levels in patients with established CKD was effective in slowing the progression of CKD as compared to that of controls. However, no significant beneficial effect of allopurinol treatment was observed on hypertension and cardiovascular outcomes in patients with CKD.

We have no financial interests related to the material in the manuscript.

\section{References}

1. Edwards NL (2008) The role of hyperuricemia and gout in kidney and cardiovascular disease. Cleve Clin J Med 75 (Suppl 5): 13-16.

2. Mazzali M, Kanellis J, HanL, Feng L, Xia YY, et al. (2002) Hyperuricemia induces a primary renal arteriolopathy in rats by a blood pressure independent mechanism. Am J Physiol Renal Physiol 282(6): 991-997.

3. Nakagawa T, Tuttle KR, Short RA, Johnson RJ (2005) Fructose induced hyperuricemia as a causal mechanism for the epidemic of the metabolic syndrome. Nat Clin Pract Nephrol 1(2): 80-86.

4. Davies Jr NS (1897) The cardiovascular and renal relations and manifestations of gout. J Am Med Assoc 29(6): 261-262.

5. Johnson RJ, Kang DH, Feig D, Kivlighn S, Kanellis J, et al. (2003) Is there a pathogenetic role for uric acid in hypertension and cardiovascular and renal disease? Hypertension 41(6): 1183-1190.
6. Mazzali M, Hughes J, Kim YG, Jefferson JA, Kang DH, et al. (2001) Elevated uric acid increases blood pressure in the rat by a novel crystal-independent mechanism. Hypertension 38(5): 1101-1106.

7. Kao MP, Ang DS, Gandy SJ, Nadir MA, Houston JG, et al. (2011) Allopurinol benefits left ventricular mass and endothelial dysfunction in chronic kidney disease. J Am Soc Nephrol 22(7): 1382-1389.

8. Goicoechea M, de Vinuesa SG, Verdalles U, Ruiz-Caro C, Ampuero J, et al. (2010) Effect of allopurinol in chronic kidney disease progression and cardiovascular risk. Clin J Am Soc Nephrol 5(8): 13881393.

9. Pai BH, Swarnalatha G, Ram R, Dakshinamurty KV (2013) Allopurinol for prevention of progression of kidney disease with hyperuricemia. Indian J Nephrol 23(4): 280-286.

10. Diller PM, Smucker DR, David B, Graham RJ (1999) Congestive heart failure due to diastolic or systolic dysfunction. Frequency and patient characteristics in an ambulatory setting. Arch Fam Med 8(5): 414-420.

11. Feig DL, Soletsky B, Johnson RJ (2008) Effect of allopurinol on the blood pressure of adolescents with newly diagnosed essential hypertension: a randomized trial. JAMA 300(8): 924-932.

12. Siu YP, Leung KT, Tong MK, Kwan TH (2006) Use of allopurinol in slowing the progression of renal disease through its ability to lower serum uric acid level. Am J Kidney Dis 47(1): 51-59.

13. Weiner DE, Tighiouart H, Elsayed EF, Griffith JL, Salem DN, et al. (2008) Uric acid and incident kidney disease in the community. J Am Soc Nephrol 19(6): 1204-1211.

14. Sundstrom J, Sullivan L, D'Agostino R, Levy D, Kannel WB, et al. (2005) Relations of serum uric acid to longitudinal blood pressure tracking and hypertension incidence. Hypertension 45(1): 28-33. 